\title{
MODELING APPROACHES TO THE DYNAMICS OF HYDROGEL SWELLING
}

By

M.C. Carme Calderer

B. Chabaud

S. Lyu

and

H. Zhang

IMA Preprint Series \# 2189

( February 2008)

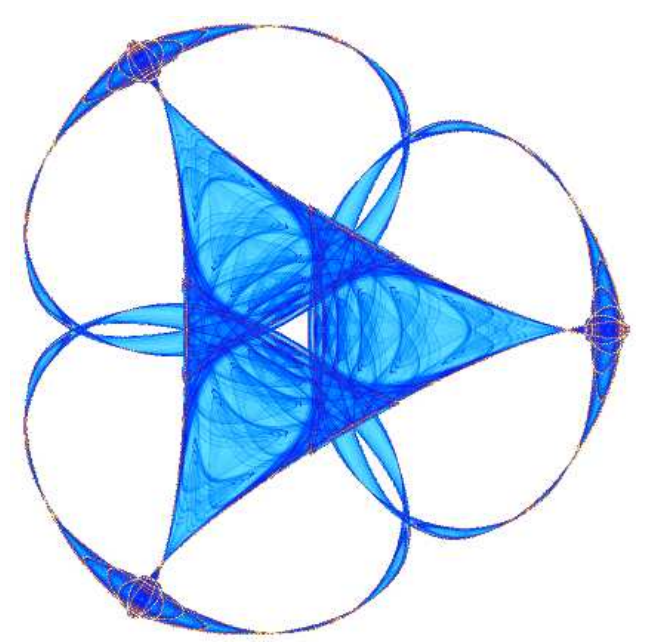

INSTITUTE FOR MATHEMATICS AND ITS APPLICATIONS

UNIVERSITY OF MINNESOTA 400 Lind Hall

207 Church Street S.E.

Minneapolis, Minnesota 55455-0436

Phone: 612-624-6066 Fax: 612-626-7370

URL: http://www.ima.umn.edu 


\title{
Modeling approaches to the dynamics of hydrogel swelling
}

\author{
M.C. Calderer, B. Chabaud \\ School of Mathematics \\ University of Minnesota \\ Minneapolis, MN 55455 \\ S. Lyu \\ Materials and Biosciences Center \\ Medtronic Inc. \\ Minneapolis, MN 55432 \\ and \\ H. Zhang \\ Department of Mathematics \\ Michigan State University \\ East Lansing, MI 48824
}

February 10, 2008

\begin{abstract}
We consider a gel as an immiscible mixture of polymer and solvent, and derive governing equations of the dynamics. They include the balance of mass and linear momentum of the individual components. The model allows to account for nonlinear elasticity, viscoelasticity, transport and diffusion. The total free energy of the system combines the elastic contribution of the polymer with the Flory-Huggins energy of mixing. The system is also formulated in terms of the center of mass velocity and the diffusive velocity, involving the total and the relative stresses. This allows for the identification of special regimes, such as the purely diffusive and the transport ones. We also obtain an equation for the rate of change of the total energy yielding decay for special choices of boundary conditions. The energy law motivates the Rayleghian variational approach discussed in the last part of the article.

We consider the case of a gel in a one-dimensional strip domain in order to study special features of the dynamics, in particular, the early dynamics. We find that the monotonicity of the extensional stress is a necessary condition to guarantee the propagation of the swelling interface between the gel and its solvent. Such monotonicity
\end{abstract}


condition is satisfied for data corresponding to linear entangled polymers. However, for polyssacharide gels the monotonicity of the stress fails at a critical volume fraction, suggesting the onset of de-swelling. The weak elasticity is responsible for the loss of monotonicity of the stress. The analysis also suggests that type II diffusion is a hyperbolic phenomenon rather than a diffusive one.

One goal is to compare the derivation method, assumptions and resulting equations with other models available in the literature, and determine their regimes of validity. The stress-diffusion coupling model by Yamaue and Doi [26] is one main benchmark. We assume that the gel is non-ionic, and neglect thermal effects.

\section{Introduction}

In this article we derive a model of the dynamics of gels from the point of view of the theory of mixtures. One goal is to compare the derivation method, assumptions and resulting equations with other models available in the literature, and determine their regimes of validity. The selection of constraints plays a main role in our development. The free energy of the model consists of the Flory-Huggins mixing energy together with an isotropic nonlinearly elastic contribution. The model accounts for viscoelastic behavior of the polymer yielding a fully nonlinear reversible stress on time-independent deformations. We also derive equations for distinctive regimes of gel behavior, namely, a purely diffusive case, and the case when only transport is present with no diffusion taking place. We list physically realistic boundary conditions that make the governing system mathematically well posed, and describe the free boundary evolution for special geometries. In particular, we find a condition for propagation of one-dimensional swelling interfaces in terms of the monotonicity of the stress-strain relation, and interpret the break-down of such a condition as the onset of de-swelling. We show that the Second Law of Thermodynamics yields an energy dissipation inequality for the total energy of the system. This inequality motives the definition of Rayleghian of the system that, in turn, yields a variational principle to derive governing equations. This is, in fact, the method employed by Yamaue and Doi in deriving the stress-diffusion coupling model ([25], [26], [27] and [10]). We assume that the gel is non-ionic, and neglect thermal effects [28].

We postulate laws of balance of mass and linear momentum for a two-component system, solvent and elastic solid. The free energy is of the Flory-Huggins type with nonlinearly elastic and isotropic stored energy function ([11], [1], [18] and [19]). We include action-reaction forces between polymer and solvent particles acting as friction forces. We postulate the Second Law of Thermodynamics in the form of the ClausiusDuhem inequality, accounting for the appropriate constraints. This yields information on the form of the constitutive equations, in particular, the dependence of the reversible components of the stress on derivatives of the free energy, the friction forces as depending on the relative velocity, and the residual inequality to be satisfied by the dissipative part of the stress.

The polymer and solvent in the gel form an immiscible mixture since the FloryHuggins free energy depends explicitly on volume fractions. We recall that a mixture is miscible if the corresponding equations do not allow to distinguish between components, that is, they are independent of volume fractions [24]. Another characterization of mixture is in terms of the compressibility of its components, which is formulated in 
terms of its component densities. For this, it is relevant to distinguish between the bulk density, $\rho$, and the true or intrinsic density, $\gamma$, of each component, the first one representing the mass of the component per unit volume of mixture, and the second one the mass per unit volume of the component, that is the density of the material in its isolated state. The ratio between the two densities defines the volume fraction, $\phi=\gamma \rho^{-1}$. A component is incompressible if its intrinsic density, $\gamma$ is constant.

The equations of balance of mass and linear momentum provide two scalar equations for the densities, $\rho_{1}$ and $\rho_{2}$ and two vector equations for the velocity fields $\mathbf{v}_{1}$ and $\mathbf{v}_{2}$, respectively. A third scalar equation is given by the constraint that the sum of volume fractions adds up to one (19), since no third material component or void are present. Altogether, in the case of a compressible mixture, these equations are not sufficient to determine the independent fields $\left\{\rho_{1}, \rho_{2}, \phi_{1}, \phi_{2}, \mathbf{v}_{1}, \mathbf{v}_{2}\right\}$ of the problem. A closure condition is needed for the problem to be mathematically well-posed. The choice of such an additional equation is a main challenge arising in the modeling of swelling systems where compressibility of the components is a relevant property of the material, such as in the case of clays ([17], [2] and [3]).

The situation in polymeric gels is somehow different since a natural candidate to closure condition is the incompressibility of the solvent. Accordingly, we set $\phi_{2}=\gamma_{2}$ in our study. The resulting system consists of equations (5), (10), (7), (8) and (19) for the fields $\left\{\rho_{1}, \phi_{1}, \phi_{2}, \mathbf{v}_{1}, \mathbf{v}_{2}\right\}$. Moreover, in this article, we mainly focus in the case that the polymer is also incompressible, and further set $\rho_{1}=\phi_{1}$. This is a reasonable assumption for many types of linear entangled polymers, and it precludes the wellknown mathematical difficulties related to the formation of shocks ([7]). The fields of the problem now include the pressure $p$ instead of the density $\rho_{1}$. We point out that the assumption of polymer incompressibility might not be appropriate near the threshold of a volume phase transition where the system may become highly compressible [20].

In section 2, we proceed to derive the governing equations of the incompressible mixture. The constraint (19), in addition to bringing a Lagrange multiplier, $p$, into the constitutive equations of the stress and of the diffusive forces, it establishes a relation between the laws of balance of mass of the components, reducing them to one single equation in the system. Such an equation can be presented in three different forms: the Eulerian equation of balance of mass of the polymer (12), the one for the solvent (13), and the Lagrangian form for the polymer (17). The latter can also be viewed as a constraint on the variables $F$, the polymer gradient of deformation matrix, and $\phi_{1}$, allowing for the elimination of $\phi_{1}$ from the system, with the model becoming purely mechanical. The choice between these forms depends on the initial and boundary conditions of the problem at hand.

Mathematically, in the case that the polymer viscosity is Newtonian, the system is of mixed hyperbolic and parabolic type. A special challenge comes from the fact that that the system combines the Eulerian description of fluids with the Lagrangian one of solids. This results into the chain rule equation relating the time derivative of the deformation gradient with the spatial gradient of velocity (71). The analysis of the latter equation is presented in the article by P.L. Lions and J.R. DiPerna [8]. Our model bears analogies with that studied by Liu and Walkington, for a mixture of elastic particles in a viscous fluid. However, the latter does not involve diffusion effects [16].

In section 3, we study the energy of the system and derive the equations of the relaxation part of the stress tensor of the polymer. This consists of a nonlinear elastic 
Cauchy contribution together with osmotic pressure coming from the mixing energy. The stress tensor of the fluid involves the corresponding osmotic pressure; in addition, both stress tensor involve the hydrostatic pressure due to the saturation constraint. In section 4 , we study the energy decay of the system. The formulation of the viscoelastic stress of the polymer is presented in section 5. We formulate the Kaye-BKZ model for gels since it yields the (elastic-osmotic) relaxation stress at the equilibrium limit.

The balance laws for the components of the mixture yield a system of equations for the gel, as presented in section 6. Specifically, they give equations of balance of linear momentum of the center of mass velocity $\mathbf{V}$, and the relative velocity $\mathbf{U}$, together with one of the equations of conservation of mass. Whereas reference to individual components may be avoided in dealing with bulk phenomena, their separate roles reemerge on the boundary, with either the solvent component or the solid one becoming relevant, according to the boundary conditions.

From the governing system, we obtain equations for special regimes. One of the regimes is purely diffusive, with no net transport taking place, such as the case of swelling of a gel in its own solvent. A system with only transport and no diffusion also results from the equations. For instance, a gel subject to a shearing flow and with non-permeable boundary. The mixture behaves as a single material with elastic and viscous properties. The problem becomes mathematically analogous to viscoelastic flow models analyzed in [15].

In the one-dimensional geometry corresponding to a strip domain, and neglecting viscous stress (by a scaling argument, this corresponds to addressing the early dynamics, rather than the better understood relaxation regimes), the system is of hyperbolic type with a dissipative source term depending on the diffusive velocity. The extensional stress is given by the function $G(\phi)$; the problem is mathematically well-posed provided $G$ is monotonically decreasing (increasing when regarded as a function of the elastic deformation). In particular, this guarantees the propagation of the swelling interface separating gel from pure solvent [6]. We find that the monotonicity property of the stress holds for the polymer data, whereas loss of monotonicity occurs in polysaccharide. Specifically, we found an interval of volume fraction in a high swelling regime, where the stress changes monotonicity. This may suggest the onset of de-swelling or a volume phase transition taking place. The weak elasticity is responsible for the lose of monotonicity of the stress. From a related point of view, the study of the early dynamics of a dry polymer slab as it comes into contact with its own solvent, shows that type II diffusion is a hyperbolic phenomena, rather than a diffusion process as its name would otherwise indicate $([6],[23])$.

In the last part of section 6 , we explain how the model that we derive relates to the quasi-static Stress-diffusion coupling model presented by Yamaue and Doi, and analyzed in some of their articles ([25], [26] and [27]). Omitting mass inertia terms and the FloryHuggins contribution to the energy yields the latter; in their applications, they further take the linear version of the model with equations that include the elastic equilibrium equation of the gel and Darcy's law for the solvent. This generalizes earlier work by Tanaka and Filmore [22] based on the elasticity equation. The stress-diffusion coupling model, in turn, results from modeling work by Doi [9] based on a variational principle for the Rayleghian. We conclude the section illustrating such a derivation in the case that the functional includes the constraint of the model and the mixing energy. Although both approaches lead to the same system of equations, one advantage of the method 
based on the Second Law of Thermodynamics is that it readily gives the constitutive equations of the reversible regime.

\section{Balance of mass, transport equations and con- straints}

We assume that the components of the gel occupy domains $\Omega_{a} \subset \mathbf{R}^{3}, a=1,2$ in the reference (Lagrangian) configuration, with $\alpha$ representing the reference volume fraction of polymer. Here the sub-indices 1 and 2 refer to polymer and solvent, respectively. The Lagrangian domains $\Omega_{a}, a=1,2$ may be distinct, since the polymer and fluid occupy different locations in space, previous to mixing. However, both components share a common domain, $\Omega$, in the Eulerian representation of the gel. The variables $\mathbf{X} \in \Omega_{a}$ and $\mathbf{x} \in \Omega$ represent Lagrangian and Eulerian coordinates, respectively. The deformation maps of polymer and solvent are given by sufficiently smooth invertible functions

$$
\begin{aligned}
& \mathbf{x}=\mathcal{M}(\mathbf{X}, t), \quad \mathbf{X} \in \Omega_{1}, \\
& \mathbf{x}=\mathcal{N}(\mathbf{X}, t), \quad \mathbf{X} \in \Omega_{2}
\end{aligned}
$$

with $F=\nabla_{\mathbf{X}} M(\mathbf{X}, t)$ denoting the gradient of deformation of the polymer. The material velocities of polymer and solvent, respectively,

$$
\begin{gathered}
\tilde{\mathbf{v}}_{1}(\mathbf{X}, t)=\frac{\partial \mathcal{M}}{\partial t}(\mathbf{X}, t), \quad \mathbf{X} \in \Omega_{1} \\
\tilde{\mathbf{v}}_{2}(\mathbf{X}, t)=\frac{\partial \mathcal{N}}{\partial t}(\mathbf{X}, t) \quad \mathbf{X} \in \Omega_{2} .
\end{gathered}
$$

The corresponding velocity fields are

$$
\mathbf{v}_{1}(\mathbf{x}, t)=\tilde{\mathbf{v}}_{1}\left(\mathcal{M}^{-1}(\mathbf{x}, t), t\right), \quad \mathbf{v}_{2}(\mathbf{x}, t)=\tilde{\mathbf{v}}_{2}\left(\mathcal{N}^{-1}(\mathbf{x}, t), t\right) .
$$

We let $\mathcal{T}_{1}(\mathbf{x}, t)$ and $\mathcal{T}_{2}(\mathbf{x}, t)$ denote Cauchy stress tensors. Each one includes elastic and dissipative contributions. We also take into account friction forces $\mathbf{f}_{a}$ per unit volume that the polymer exerts upon the fluid, and vice-verse.

\subsection{Governing equations for a mixture of incompressible solvent and compressible polymer}

According to the theory of mixtures, polymer and solvent particles may occupy the same location, with volume fractions $\phi_{1}(\mathbf{x}, t), \phi_{2}(\mathbf{x}, t)$, respectively. We also assume that no other material is present in the region, so

$$
\phi_{1}(\mathbf{x}, t)+\phi_{2}(\mathbf{x}, t)=1
$$

holds. We let $\rho_{1}$ and $\rho_{2}$ denote the mass densities of the components per unit volume in space. These are related to the true or intrinsic densities ( $\left.\frac{\text { mass of component }}{\text { volume of component }}\right), \gamma_{1}$ and $\gamma_{2}$ as follows:

$$
\rho_{1}=\gamma_{1} \phi_{1}, \quad \rho_{2}=\gamma_{2} \phi_{2} .
$$


The volume fractions $\phi_{1}$ and $\phi_{2}$ are independent fields of the constitutive equations postulated later, and consequently, the mixture is immiscible [24]. The local forms of the laws of balance of mass and linear momentum in Eulerian coordinates are given by

$$
\begin{aligned}
& \frac{\partial \rho_{1}}{\partial t}+\left(\mathbf{v}_{1} \cdot \nabla\right) \rho_{1}+\rho_{1} \nabla \cdot \mathbf{v}_{1}=0 \\
& \frac{\partial \rho_{2}}{\partial t}+\left(\mathbf{v}_{2} \cdot \nabla\right) \rho_{2}+\rho_{2} \nabla \cdot \mathbf{v}_{2}=0 \\
& \rho_{1} \frac{\partial \mathbf{v}_{1}}{\partial t}+\rho_{1}\left(\mathbf{v}_{1} \cdot \nabla\right) \mathbf{v}_{1}=\nabla \cdot \mathcal{T}_{1}+\mathbf{f}_{1} \\
& \rho_{2} \frac{\partial \mathbf{v}_{2}}{\partial t}+\rho_{2}(\mathbf{v} \cdot \nabla) \mathbf{v}_{2}=\nabla \cdot \mathcal{T}_{2}+\mathbf{f}_{2}
\end{aligned}
$$

The independent fields of the problem are $\left\{\rho_{1}, \rho_{2}, \phi_{1}, \phi_{2}, \mathbf{v}_{1}, \mathbf{v}_{2}\right\}\left(\mathcal{T}_{1}, \mathcal{T}_{2}, \mathbf{f}_{1}\right.$ and $\mathbf{f}_{2}$ will be given in terms of the former). We observe that the previous system together with (4) is not sufficient to determine all the independent variables since one additional scalar equation is needed. Therefore, one closure condition should be added to the system to make it well-posed. There is no general physical law dictating such a condition and, in some cases, the criterion is to recover well known phenomenological relations of the theory. For instance, this is the case of Law's equation in porous media [17], [2] and [3]. Another choice found in the literature is to require that the total equilibrium stress is zero which leads to a relation between the pressure part of the stress tensors. The resulting equations which hold at equilibrium only justify the validity of the linear theory.

In our case, it is reasonable to assume that the solvent is incompressible, leading to the relation $\gamma_{2}=1$. (For simplicity, we take the constant intrinsic density to be 1 ), and consequently, the system (4)-(8) is well posed. Note that in such a case, the governing system does not involve any Lagrange multipliers. This is indeed the case of compressible elasticity, and it is consistent with the fact that $\gamma_{1}$ is allowed to be free. On the other hand, it does not correspond to the gel models by Doi and Yamaue in which the Lagrange multiplier plays a prominent role. We argue that the additional constraint of polymer incompressibility is at play. We distinguish two cases according to this observation.

The balance laws in the case of incompressible solvent and compressible polymer consist of (4)-(8) but replacing $\rho_{2}$ with $\phi_{2}$ in (6) The constitutive equations for $\mathcal{T}_{i}$ and $\left\{i, i=1,2\right.$, depend on the fields $\left\{\rho_{1}, \phi_{1}, \phi_{2}, \mathbf{v}_{1}, \mathbf{v}_{2}\right\}$. Moreover, using equation (4) to eliminate $\phi_{2}$, we are left with a governing system for the variables $\left\{\rho_{1}, \phi_{1}, \mathbf{v}_{1}, \mathbf{v}_{2}\right\}$ consisting of (5)-(8) with equation (6) replaced with

$$
\left.\frac{\partial \phi_{1}}{\partial t}-\nabla \cdot\left(\left(1-\phi_{1}\right) \mathbf{v}_{2}\right)\right)=0
$$

The assumption of polymer compressibility may be justified in the following situations:

1. Experiments involving volume transitions in gels suggest a highly compressible regime near the critical volume fraction $\phi_{c}$ where the first order transition takes place. The transition from a swollen regime to a collapsed one (or viceversa) may involve a significant expansion (or compression) of the polymer.

2. Incompressibility is itself an ideal property; indeed, it may be more realistic to think of the polymer as being almost incompressible. The incompressible regime arises then as a limit of almost incompressible ones. This is a condition that we will formulate in terms of the free energy. 
Away from the phase transition, we may then consider the polymer as being incompressible with compressibility manifesting itself at the transition volume fraction.

\subsection{Governing equations for a mixture of incompressible solvent and polymer}

For simplicity, we take $\gamma_{1}=\gamma_{2}=1$. In this case, the densities and volume fractions coincide, and hereforth we will only refer to the latter,

$$
\rho_{1}=\phi_{1}, \quad \rho_{2}=\phi_{2}
$$

We point out that incompressibility of mixture components allows for deformations with Jacobian different than one. Indeed, changes in density of one component occur by changes of volume fraction. In particular, an extension of the polymer network that decreases the volume fraction $\phi_{1}$ may occur at the expense of increasing the volume fraction $\phi_{2}$.

The local forms of the laws of balance of mass and linear momentum in Eulerian coordinates are

$$
\begin{aligned}
& \frac{\partial \phi_{1}}{\partial t}+\left(\mathbf{v}_{1} \cdot \nabla\right) \phi_{1}+\phi_{1} \nabla \cdot \mathbf{v}_{1}=0 \\
& \frac{\partial \phi_{2}}{\partial t}+\left(\mathbf{v}_{2} \cdot \nabla\right) \phi_{2}+\phi_{2} \nabla \cdot \mathbf{v}_{2}=0 \\
& \phi_{1} \frac{\partial \mathbf{v}_{1}}{\partial t}+\phi_{1}\left(\mathbf{v}_{1} \cdot \nabla\right) \mathbf{v}_{1}=\nabla \cdot \mathcal{T}_{1}+\mathbf{f}_{1} \\
& \phi_{2} \frac{\partial \mathbf{v}_{2}}{\partial t}+\phi_{2}(\mathbf{v} \cdot \nabla) \mathbf{v}_{2}=\nabla \cdot \mathcal{T}_{2}+\mathbf{f}_{2}
\end{aligned}
$$

where $\nabla \equiv \nabla_{\mathbf{x}}$ refers to gradient with respect to the Eulerian variables. The kinematic compatibility condition between the time derivative of the deformation gradient and the velocity gradient results from the chain rule:

$$
F_{t}+\left(\mathbf{v}_{1} \cdot \nabla\right) F=\left(\nabla \mathbf{v}_{1}\right) F
$$

The Lagrangian form of the equation of balance of mass of the polymer component is

$$
\begin{aligned}
\int_{\Omega} \phi_{1} d \mathbf{x} & =\int_{\Omega_{0}} \alpha d \mathbf{X} \\
& =\int_{\Omega_{0}} \phi_{1} \operatorname{det} F d \mathbf{x}
\end{aligned}
$$

where $0 \leq \alpha \leq 1$ denotes the volume fraction of polymer in the reference configuration. We assume that it is satisfied for all parts of the body $\Omega_{0}$, yielding the local constraint

$$
\phi_{1} \operatorname{det} F=\alpha .
$$

The latter relation is equivalent to equation (12). Defining the center of mass velocity

$$
\mathbf{V}=\sum_{a=1,2} \phi_{a} \mathbf{v}_{a}
$$


it follows that addition of equations (12) and (13), taking (4) into account yield

$$
\nabla \cdot \mathbf{V}=\sum_{a} \nabla \cdot\left(\phi_{a} \mathbf{v}_{a}\right)=\sum_{a} \mathbf{v}_{a} \cdot \nabla \phi_{a}+\phi_{a} \nabla \cdot \mathbf{v}_{a}=0
$$

This suggests formulating the governing system as consisting of equations (4), (14), (15) and one of the following additional sets:

- equations (12) and (13),

- equations (12) and (19), or (13) and (19),

- equations (17) and (19).

Remark. A special case of mathematical interest arises by adding an additional constraint to the system, that is, requiring that the fluid component can only experience isochoric deformations,

$$
\nabla \cdot \mathbf{v}_{2}=0 .
$$

It is easy to see that in one-dimensional geometry, the only change in volume fraction resulting from such dynamics is a purely hyperbolic, in the sense that the initial volume fraction profile travels through the domain undisturbed. However, in two and three dimensional geometries, it may still allow for non-trivial swelling dynamics. It is also an appropriate model of how rheological properties of the polymer depend on the amount of solvent held by the polymer.

The previous set of equations do not yet fully determine the system, since constitutive equations and additional kinematic relations need to be prescribed.

We now formulate the balance of energy. Letting $\varepsilon_{a}, a=1,2$ denote component internal energy densities, we write the equation of balance of energy

$$
\sum_{a}\left(\phi_{a} \frac{\partial \varepsilon_{a}}{\partial t}+\phi_{a}\left(\mathbf{v}_{a} \cdot \nabla\right) \varepsilon_{a}\right)=\sum_{a}\left(\operatorname{tr}\left(\nabla \mathbf{v}_{a} \mathcal{T}_{a}\right)+\nabla \cdot \mathbf{q}_{a}+\phi_{a} r_{a}+\hat{\varepsilon}_{a}\right)
$$

Here $\hat{\varepsilon}_{a}(\mathbf{x}, t)$ represent the internal energy rate production in each component, $\mathbf{q}_{a}, a=$ 1,2 the heat flux vectors and $r_{a}$ radiation terms. The friction forces and the internal production rate quantities are required to satisfy

$$
\begin{aligned}
& \mathbf{f}_{1}=-\mathbf{f}_{2} \\
& \sum_{a=1,2} \hat{\varepsilon}_{a}(\mathbf{x}, t)+\left(\mathbf{v}_{a}-\mathbf{V}\right) \cdot \mathbf{f}_{a}=0 .
\end{aligned}
$$

The free energy density and the entropy densities of the mixture, respectively, can be written in terms of their components as follows:

$$
\begin{aligned}
& \Psi=\phi_{1} \psi_{1}\left(\phi_{1}, \phi_{2}, F\right)+\phi_{2} \psi_{2}\left(\phi_{1}, \phi_{2}\right) \\
& \eta=\phi_{1} \eta_{1}\left(\phi_{1}, \phi_{2}, F\right)+\phi_{2} \eta_{2}\left(\phi_{1}, \phi_{2}\right) .
\end{aligned}
$$

Moreover, we will make use of the relations

$$
\psi_{a}=\varepsilon_{a}-T_{a} \eta_{a}
$$

defining the free energy density $\psi_{a}$ of the components in terms of the corresponding internal energy $\varepsilon_{a}$ and entropy $\eta_{a} ; T_{a}$ denotes absolute temperature. The free energy $\Psi$ 
of the system accounts for the Flory-Huggins and elastic contributions given in the next subsection.

Remark. It may seem more reasonable to directly postulate $\Psi$ rather than appealing to components $\psi_{a}, a=1,2$ as in (24), and likewise for the entropy of the system. For a justification of our approach, we refer to [12].

Expressions of the component free energies [12] that yield (47) are

$$
\begin{aligned}
& \psi_{1}=\frac{K_{B} T}{2 V_{m}} \chi \phi_{2}^{2}+\frac{K_{B} T}{N_{1} V_{m}} \log \phi_{1}+W(F), \\
& \psi_{2}=\frac{K_{B} T}{2 V_{m}} \chi \phi_{1}^{2}+\frac{K_{B} T}{N_{2} V_{m}} \log \phi_{2} .
\end{aligned}
$$

\subsection{Thermodynamics and constitutive equations}

We now postulate the second law of thermodynamics for the mixture,

$$
\sum_{a=1,2} \phi_{a} \dot{\eta}_{a}-\nabla \cdot\left(\frac{\mathbf{q}_{a}}{T_{a}}\right)-\phi_{a} \frac{r_{a}}{T_{a}} \geq 0
$$

Substituting (26) into (29) and the balance of energy [21], we get

$$
\sum_{a} \frac{1}{T_{a}}\left\{\operatorname{tr}\left(\mathcal{T}_{a}^{T} \nabla \mathbf{v}_{a}\right)-\dot{T}_{a} \phi_{a} \eta_{a}-\phi_{a} \dot{\psi}_{a}+\frac{1}{T_{a}} \mathbf{q}_{a} \cdot \nabla T_{a}-\mathbf{f}_{a} \cdot\left(\mathbf{v}_{a}-\mathbf{V}\right)\right\} \geq 0
$$

From now on, we will neglect thermal effects and consider isothermal regimes only, so that the previous inequality expresses mechanical dissipation,

$$
\sum_{a} \operatorname{tr}\left(\mathcal{T}_{a}^{T} \nabla \mathbf{v}_{a}\right)-\phi_{a} \dot{\psi}_{a}-\mathbf{f}_{a} \cdot \mathbf{v}_{a} \geq 0
$$

We now reformulate the entropy inequality when the fields satisfy the constraint (19). The following relations, based on the chain-rule, will be used in simplifying the entropy inequality:

$$
\begin{aligned}
& \nabla \mathbf{v}_{1}=\dot{F} F^{-1} \\
& \nabla \cdot \mathbf{v}_{1}=\operatorname{tr}\left(\nabla \mathbf{v}_{1}\right)=\operatorname{tr}\left(F^{-1} \dot{F}\right) .
\end{aligned}
$$

Let $p$ denote the Lagrange multiplier associated with constraint (19). We argue that the dissipation inequality should hold for those processes such that the constraints are approximately satisfied,

$$
\sum_{a} \operatorname{tr}\left(\mathcal{T}_{a}^{T} \nabla \mathbf{v}_{a}\right)-\phi_{a} \dot{\psi}_{a}-\mathbf{f}_{a} \cdot \mathbf{v}_{a}-p \nabla \cdot\left(\phi_{a} \mathbf{v}_{a}\right) \geq 0
$$

Using (32), the latter can be written as follows

$$
\begin{aligned}
& \operatorname{tr}\left\{\left(F^{-1} \mathcal{T}_{1}^{T}+\phi_{1}\left(\phi_{1} \frac{\partial \psi_{1}}{\partial \phi_{1}}+\phi_{2} \frac{\partial \psi_{2}}{\partial \phi_{1}}\right) F^{-1}-\phi_{1}{\left.\left.\frac{\partial \psi_{1}}{\partial F}+p \phi_{1} F^{-1}\right) \dot{F}\right\}}^{T}+\operatorname{tr}\left\{\left(\mathcal{T}_{2}^{T}+\phi_{2}\left(\phi_{1} \frac{\partial \psi_{1}}{\partial \phi_{2}}+\phi_{2} \frac{\partial \psi_{2}}{\partial \phi_{2}}+p\right) I\right) \nabla \mathbf{v}_{2}\right\}\right.\right. \\
& -\left(\mathbf{f}_{1}-p \nabla \phi_{1}\right) \cdot \mathbf{v}_{1}-\left(\mathbf{f}_{2}-p \nabla \phi_{1}\right) \cdot \mathbf{v}_{2} \geq 0 .
\end{aligned}
$$


Requiring the inequality to be satisfied for all choices $F, \dot{F}, \phi_{1}, \phi_{2}, \mathbf{v}_{1}-\mathbf{v}_{2}, \nabla \mathbf{v}_{2}$ yields the forms of $\mathcal{T}_{a}$, of the reversible contributions to the stress tensor,

$$
\begin{aligned}
& \mathcal{T}_{1}^{r}=\phi_{1} \frac{\partial \psi_{1}}{\partial F} F^{T}-\left(\phi_{1} p+\pi_{1}\right) I \\
& \mathcal{T}_{2}^{r}=-\left(\phi_{2} p+\pi_{2}\right) I
\end{aligned}
$$

where

$$
\begin{array}{ll}
\pi_{1}= & \phi_{1}\left(\phi_{1} \frac{\partial \psi_{1}}{\partial \phi_{1}}+\phi_{2} \frac{\partial \psi_{2}}{\partial \phi_{1}}\right), \\
\pi_{2}= & \phi_{2}\left(\phi_{1} \frac{\partial \psi_{1}}{\partial \phi_{2}}+\phi_{2} \frac{\partial \psi_{2}}{\partial \phi_{2}}\right)
\end{array}
$$

denote the mixing components of the osmotic pressure. Substituting (35) and (36) into (34), the residual inequality for $\mathbf{f}_{a}$, and the dissipative parts $\mathcal{T}_{1}^{d}$ and $\mathcal{T}_{2}^{d}$ of the stress tensors follows:

$$
-\operatorname{tr}\left(\dot{F}^{T} \mathcal{T}_{1}^{d} F^{-T}+\left(\nabla \mathbf{v}_{2}\right)^{T} \mathcal{T}_{2}^{d}\right)+\sum_{a}\left(\mathbf{f}_{a}-p \nabla \phi_{a}\right) \cdot \mathbf{v}_{a} \leq 0
$$

Using once more the arbitrariness in the choice of $\dot{F}$ and $\nabla \mathbf{v}_{2},\left(\mathbf{v}_{1}-\mathbf{v}_{2}\right)$, we get

$$
\left(\mathbf{f}_{1}-p \nabla \phi_{1}\right) \cdot\left(\mathbf{v}_{1}-\mathbf{v}_{2}\right) \leq 0
$$

In order for the previous inequality to hold, we take

$$
\mathbf{f}_{1}=p \nabla \phi_{1}-\beta\left(\mathbf{v}_{1}-\mathbf{v}_{2}\right), \quad \mathbf{f}_{2}=p \nabla \phi_{2}+\beta\left(\mathbf{v}_{1}-\mathbf{v}_{2}\right),
$$

where $\beta=\beta\left(\phi_{1}, \phi_{2}\right)>0$ is related to the diffusion coefficient in Darcy's law as shown later. Using (40), inequality (39) finally yields,

$$
\begin{aligned}
& \operatorname{tr}\left(\dot{F}^{T} \mathcal{T}_{1}^{d} F^{-T}\right) \geq 0 \\
& \operatorname{tr}\left(\left(\nabla \mathbf{v}_{2}\right)^{T} \mathcal{T}_{2}^{d}\right) \geq 0 .
\end{aligned}
$$

Remark. In viscoelastic gels, the stress $\mathcal{T}_{1}$ depends on the past history of the deformation gradient. In such cases, the reversible stress component corresponds to the relaxation limit of of $\mathcal{T}_{1}$, with the dissipative component, being the difference between the total stress and its relaxation limit.

Remark. For the mixture with incompressible solvent and compressible polymer, the equations for the equilibrium part of the stress tensor are given by

$$
\begin{aligned}
& \mathcal{T}_{1}=\rho_{1}\left(\frac{\partial \psi_{1}}{\partial F}\right) F^{T}-\rho_{1}^{2} \frac{\partial \psi_{1}}{\partial \rho_{1}} I \\
& \mathcal{T}_{2}=-\left(\rho_{1} \phi_{2}\left(\frac{\partial \psi_{1}}{\partial \phi_{2}}-\frac{\partial \psi_{1}}{\partial \phi_{1}}\right)+\phi_{2}^{2}\left(\frac{\partial \psi_{2}}{\partial \phi_{2}}-\frac{\partial \psi_{2}}{\partial \phi_{1}}\right)\right) I .
\end{aligned}
$$




\section{$3 \quad$ Elastic and mixing energies.}

The assumption that the free energy $W(F)$ is isotropic implies that

$$
W(F)=\hat{w}\left(I_{1}, I_{2}, I_{3}\right), \quad \text { with } B=F F^{T}
$$

where

$$
I_{1}=\operatorname{tr} B, \quad 2 I_{2}=\left(\operatorname{tr}^{2} B-\operatorname{tr} B^{2}\right), \quad I_{3}=\operatorname{det} B .
$$

Combining the elastic free energy of the polymer and Flory-Huggins energy of the mixture gives the total free energy (24) of the mixture

$$
\Psi=\frac{K_{B} T}{V_{m}}\left(\frac{\chi}{2} \phi_{1} \phi_{2}+\frac{1}{N_{1}} \phi_{1} \log \phi_{1}+\frac{1}{N_{2}} \phi_{2} \log \phi_{2}\right)+\phi_{1} W(F) .
$$

where

$$
W(F)=\frac{3 K_{B} T}{2 N_{x} V_{m}}\left((\operatorname{det} B)^{\frac{1}{3}}-1-\frac{1}{6} \log (\operatorname{det} B)\right)+\mu w\left(I_{1}, I_{2}, I_{3}\right) .
$$

We will consider neo-Heokean elasticity so that

$$
w\left(I_{1}, I_{2}, I_{3}\right)=\operatorname{tr} B
$$

The parameters of the previous equations correspond to the following:

- $V_{m}$ is the volume occupied by one monomer;

- $K_{B}$ is the Boltzmann constant, and $T$ is the absolute temperature;

- $N_{1}, N_{2}$ denote the number of lattice sites occupied by the polymer and the solvent, respectively.

- $N_{x}$ is the number of monomers between entanglement points;

- $\chi$ is the Flory interaction parameter;

- $\beta$ is the polymer drag coefficient;

- $\mu$ is a scaling parameter related to the shear modulus.

Parameter values appropriate to semi-dry polymers [4] are given next.

$$
\left|\begin{array}{ll}
N_{x} & 20 \\
N_{1} & 1000 \\
N_{2} & 1 \\
V_{m} & .1 \mathrm{n} m^{3} \\
\chi & .5 \\
\mu & 2 \times 10^{2} 2 \times 10^{6} \frac{\mathrm{pN}}{\mathrm{nm} m^{2}} \\
\beta & 2.4 \times 10^{10} \frac{\mathrm{pNs}}{\mathrm{nm}^{4}} \\
T & 300 \mathrm{degreesK}
\end{array}\right|
$$

Parameter values for polysaccharides [5] are as in the previous table with the following exceptions:

$$
\left|\begin{array}{ll}
\mu & 2 \times 10^{-5} \frac{\mathrm{pN}}{\mathrm{nm}^{2}} \\
\beta & 2.4 \times 10^{3} \frac{\mathrm{pNs}}{\mathrm{nm}^{4}}
\end{array}\right|
$$


We conclude this subsection by calculating the expressions of the stress tensor.

$$
\frac{\partial W(F)}{\partial F}=\frac{K_{B} T}{N_{x} V_{m}}\left((\operatorname{det} F)^{-\frac{1}{3}}-\frac{1}{2 \operatorname{det} F}\right) \operatorname{adj}(F)+2 \mu F,
$$

where adj $F$ denotes the adjoint of $F$. Moreover,

$$
\frac{\partial W(F)}{\partial F} F^{T}=\frac{K_{B} T}{V_{m} N_{x}}\left((\operatorname{det} F)^{\frac{2}{3}}-\frac{1}{2}\right) \mathbf{I}+2 \mu F F^{T} .
$$

We point out that the terms multiplying the identity matrix are elastic contributions to the osmotic pressure, which we denote

$$
\Pi_{1}=\pi_{1}+\frac{K_{B} T}{N_{x} V_{m}}\left(\frac{1}{2}-(\operatorname{det} B)^{\frac{1}{3}}\right), \Pi_{2}=\pi_{2},
$$

where $\pi_{i}$ denote the mixing contributions to the osmotic pressure (37) and (38). Using the calculation (52) in equations (35) and (36), we obtain the equilibrium stresses when the polymer is a neo-Heokean solid:

$$
\begin{aligned}
& \mathcal{T}_{1}^{r}=-\left(\Pi_{1}+p \phi_{1}\right) I+2 \mu \phi_{1} B \\
& \mathcal{T}_{2}^{r}=-\left(\phi_{2} p+\Pi_{2}\right) I .
\end{aligned}
$$

We conclude this subsection with the expression of the stress tensors when the elastic stored energy function of the polymer is as in (48). A calculation as in [19] gives

$$
\begin{aligned}
\mathcal{T}_{1}^{r} & =\phi_{1} \mathcal{H}(B)-\left(\Pi_{1}+\phi_{1} p\right) I, \quad \text { with } \\
\mathcal{H} & =2 \frac{\mu}{\operatorname{det} B^{\frac{1}{2}}}\left(\frac{\partial w}{\partial I_{1}} B+\frac{\partial w}{\partial I_{2}}\left(I_{1} B-B^{2}\right)+\operatorname{det} B \frac{\partial w}{\partial I_{3}} I\right) .
\end{aligned}
$$

\section{Remark.}

We observe that substituting equations (4) and (17) into (54) and (55) allows us to obtain a fully mechanical form of the stresses, with dependence on gradient of deformation and its invariants, and on the Lagrange multipliers.

Notation. The extra stresses $\sigma_{1}$ and $\sigma_{2}$ denote the constitutive contributions in the total stress components, $\mathcal{T}_{1}$ and $\mathcal{T}_{2}$, respectively, that is

$$
\sigma_{1}=\mathcal{T}_{1}+\phi_{1} p I, \quad \sigma_{2}=\mathcal{T}_{2}+\phi_{2} p I
$$

\section{$4 \quad$ Energy Dissipation}

We now show that the rate of change of the total energy is bounded above by the work per unit time done by the boundary forces (i.e., the power of the forces acting on the boundary of the domain occupied by the gel).

Let $\Omega(t) \subset \mathbf{R}^{3}$ denote the region occupied by the gel at time $t$. Let $\nu$ represent the unit outer normal to the boundary, $\partial \Omega(t)$.

Suppose that $\mathbf{v}_{1}(\mathbf{x}, t), \mathbf{v}_{2}(\mathbf{x}, t), \phi_{1}(\mathbf{x}, t), \phi_{2}(\mathbf{x}, t)$ and $p(\mathbf{x}, t)$ is a smooth solution of the governing equations (12), (13), (14), (15), (35), (36), (24) and (4) for prescribed initial and boundary conditions. Then the following inequality holds: 


$$
\begin{aligned}
& \frac{d}{d t} \int_{\Omega(t)} \frac{1}{2}\left(\phi_{1}\left|\mathbf{v}_{1}\right|^{2}+\phi_{2}\left|\mathbf{v}_{2}\right|^{2}+\Psi\right) d \mathbf{x} \\
& \leq \int_{\partial \Omega_{t}} \mathbf{t}_{1} \cdot \mathbf{v}_{1}+\mathbf{t}_{2} \cdot \mathbf{v}_{2},
\end{aligned}
$$

where $\mathbf{t}_{1}=\mathcal{T}_{1} \nu$ and $\mathbf{t}_{2}=\mathcal{T}_{2} \nu$ and $\nu$ denotes normal to the boundary.

To derive the previous inequality, we carry out the dot product of equations (14) and (15) by $\mathbf{v}_{1}$ and $\mathbf{v}_{2}$, respectively, add up the two equations and integrate the resulting expression on $\Omega$ :

$$
\begin{aligned}
& \frac{d}{d t} \int_{\Omega(t)}\left(\frac{\phi_{1}}{2}\left|\mathbf{v}_{1}\right|^{2}+\frac{\phi_{2}}{2}\left|\mathbf{v}_{2}\right|^{2}\right) d \mathbf{x}= \\
& \int_{\Omega(t)}\left(\mathbf{v}_{1} \cdot \operatorname{div} \mathcal{T}_{1}+\mathbf{v}_{2} \cdot \operatorname{d} i v \mathcal{T}_{2}+\mathbf{v}_{1} \cdot \mathbf{f}_{1}+\mathbf{v}_{2} \cdot \mathbf{f}_{2}\right) d \mathbf{x} .
\end{aligned}
$$

Application of the divergence theorem to the terms involving $\mathcal{T}$ gives

$$
\begin{aligned}
& \frac{d}{d t} \int_{\Omega(t)}\left(\frac{\phi_{1}}{2}\left|\mathbf{v}_{1}\right|^{2}+\frac{\phi_{2}}{2}\left|\mathbf{v}_{2}\right|^{2}\right) d \mathbf{x}= \\
& -\int_{\Omega(t)} \operatorname{tr}\left(\mathcal{T}_{1}^{T}\left(\nabla \mathbf{v}_{1}\right)+\mathcal{T}_{2}^{T}\left(\nabla \mathbf{v}_{2}\right)\right) d \mathbf{x}+\int_{\Omega(t)}\left(\mathbf{v}_{1} \cdot \mathbf{f}_{1}+\mathbf{v}_{2} \cdot \mathbf{f}_{2}\right) d \mathbf{x} \\
& +\int_{\partial \Omega(t)}\left(\mathbf{t}_{1} \cdot \mathbf{v}_{1}+\mathbf{t}_{2} \cdot \mathbf{v}_{2}\right) d S
\end{aligned}
$$

Equivalently,

$$
\begin{aligned}
& \frac{d}{d t} \int_{\Omega(t)}\left(\frac{\phi_{1}}{2}\left|\mathbf{v}_{1}\right|^{2}+\frac{\phi_{2}}{2}\left|\mathbf{v}_{2}\right|^{2}\right) d \mathbf{x}-\int_{\partial \Omega(t)}\left(\mathbf{t}_{1} \cdot \mathbf{v}_{1}+\mathbf{t}_{2} \cdot \mathbf{v}_{2}\right) d S= \\
& -\int_{\Omega(t)} \operatorname{tr}\left(\mathcal{T}_{1}^{T}\left(\nabla \mathbf{v}_{1}\right)+\mathcal{T}_{2}^{T}\left(\nabla \mathbf{v}_{2}\right)\right) d \mathbf{x}+\int_{\Omega(t)}\left(\mathbf{v}_{1} \cdot \mathbf{f}_{1}+\mathbf{v}_{2} \cdot \mathbf{f}_{2}\right) d \mathbf{x} .
\end{aligned}
$$

Applying inequality (33) to the previous equation yields

$$
\begin{aligned}
& \frac{d}{d t} \int_{\Omega(t)}\left(\frac{\phi_{1}}{2}\left|\mathbf{v}_{1}\right|^{2}+\frac{\phi_{2}}{2}\left|\mathbf{v}_{2}\right|^{2}\right) d \mathbf{x}+\int_{\Omega(t)}\left(\phi_{1} \dot{\psi}_{1}+\phi_{2} \dot{\psi}_{2}\right) d \mathbf{x} \\
& -\int_{\partial \Omega(t)}\left(\mathbf{t}_{1} \cdot \mathbf{v}_{1}+\mathbf{t}_{2} \cdot \mathbf{v}_{2}\right) d S \leq 0 .
\end{aligned}
$$

By the transport theorem, the previous inequality becomes

$$
\frac{d}{d t} \int_{\Omega(t)}\left[\left(\frac{\phi_{1}}{2}\left|\mathbf{v}_{1}\right|^{2}+\frac{\phi_{2}}{2}\left|\mathbf{v}_{2}\right|^{2}\right)+\left(\phi_{1} \psi_{1}+\phi_{2} \psi_{2}\right)\right] d \mathbf{x}-\int_{\partial \Omega(t)}\left(\mathbf{t}_{1} \cdot \mathbf{v}_{1}+\mathbf{t}_{2} \cdot \mathbf{v}_{2}\right) d S \leq 0
$$

giving (59) by taking into account (24). 


\section{Viscoelasticity}

We assume that the dissipation of the gel stems mostly from the viscosity of the polymer, with the solvent contribution taken to be Newtonian. We now focus on modeling the dissipative stress of the polymer.

In the case of steady flow and small strain rate, Newtonian behavior is recovered, and the dissipative and total stress expressions are, then

$$
\begin{aligned}
& \mathcal{T}_{1}^{d}=\eta_{0} \mathbf{D}_{1}, \\
& \mathcal{T}_{1}=\mathcal{T}_{1}^{r}+\eta_{0} \mathbf{D}_{1},
\end{aligned}
$$

with $\mathbf{D}_{1}=\frac{1}{2}\left(\nabla \mathbf{v}_{1}+\left(\nabla \mathbf{v}_{1}\right)^{T}\right), \eta_{0}>0$. Likewise, for a viscous solvent, we write

$$
\begin{aligned}
& \mathcal{T}_{2}^{d}=\eta_{1} \mathbf{D}_{2}, \\
& \mathcal{T}_{2}=-\Pi_{2} I+\eta_{0} \mathbf{D}_{2} .
\end{aligned}
$$

In general $\eta_{0}>>\eta_{1}>0$ holds.

Increasing the strain rate to achieve a weak departure from Newtonian behavior yields a higher order of approximation of the stress

$$
\mathcal{T}_{1}=2 \eta_{0} \mathbf{D}_{1}-2 \epsilon \eta_{0}\left(\dot{D}_{1}-\left(\nabla \mathbf{v}_{1}\right)^{T} \mathbf{D}_{1}-\mathbf{D}_{1}\left(\nabla \mathbf{v}_{1}\right)\right)+\mathcal{T}_{1}^{r}
$$

with $\dot{\mathbf{D}}$ denoting material time derivative, and $\epsilon>0$ small. If multiple relaxation times are present, it is then appropriate to assume that the dissipative stress depends on the past history of the deformation. There are many models in the literature appropriate to specific regimes and materials, solids and fluids. Applications to viscoelastic solids in comparison to fluids, involve the additional issue of how to accurately account for, both, viscous and elastic properties. Two criteria that must be satisfied by the chosen model are:

- the viscoelastic stress yields the correct instantaneous elastic response when the deformation experiences a discontinuity in time,

- the elastic relaxation response is recovered, that is, when the deformation history is set constant, the viscoelastic stress reduces to the reversible contribution.

For this, we propose a viscoelastic constitutive equation based on the Kaye-BKZ model ([13], page 75), ([18], page 158). Such a model is based on postulating a functional of the form

$$
w(\mathbf{x}, t)=\hat{w}\left(I_{1}, I_{2}, I_{3}, t-t^{\prime}\right), I_{i}=I_{i}(\mathbf{x}, t), t>t^{\prime},
$$

and define

$$
\begin{aligned}
\mathcal{T}_{1}(\mathbf{x}, t) & =\int_{-\infty}^{t}\left(2 \operatorname{det} B\left(t, t^{\prime}\right)^{-\frac{1}{2}}\left(\hat{w}_{I} B\left(t, t^{\prime}\right)+\hat{w}_{I_{2}}\left(I_{1} B\left(t, t^{\prime}\right)-B\left(t, t^{\prime}\right)^{2}\right)+I_{3} \hat{w}_{I_{3}} \mathbf{I}\right) d t^{\prime}\right. \\
& \left.-\quad\left(\pi_{1} \phi_{1} p\right) \mathbf{I}\right)
\end{aligned}
$$

with $\pi_{1}$ as in (37). Here

$$
B\left(t, t^{\prime}\right)=F\left(t, t^{\prime}\right) F^{T}\left(t, t^{\prime}\right), \quad \text { and } \quad F\left(t, t^{\prime}\right)=\frac{\partial \mathcal{M}(\mathbf{X}, t)}{\partial \mathcal{M}\left(\mathbf{X}, t^{\prime}\right)}
$$


with $\mathbf{x}=\mathcal{M}(\mathbf{X}, t)$ as in (1).

Remark. The Kaye-BKZ model is often formulated for incompressible materials and involving the tensor $C=F^{T} F$ instead of $B$.

The dependence on past history enters through the energy functional $w$. In applications, the assumption of factorability is often made, that is,

$$
\hat{w}\left(I_{1}, I_{2}, I_{3}, s\right)=m(s) w\left(I_{1}, I_{2}, I_{3}\right),
$$

where $m() \geq$.0 and $m^{\prime}()<$.0 and $w$ is the elastic stored energy function.

In the case that the elasticity is neo-Heokean (49), and (66) holds, the stress in (65) reduces to

$$
\begin{aligned}
\mathcal{T}_{1}(\mathbf{x}, t) & =\int_{-\infty}^{t} \frac{2}{\left(\operatorname{det} B\left(t, t^{\prime}\right)\right)^{\frac{1}{2}}}\left(m\left(t-t^{\prime}\right) B\left(t, t^{\prime}\right) d t^{\prime}\right. \\
& \left.-\quad\left(\pi_{1}+\phi_{1} p\right) \mathbf{I}\right) .
\end{aligned}
$$

The viscoelastic relation in (67) is known as the Lodge model.

\section{Governing equations of gels}

We derive equations for the mixture from those for the individual components. The fields of the gel consist of

$$
\left\{\mathbf{V}, \mathbf{U}, \mathcal{T}, \phi_{2}, p\right\}
$$

where $\mathbf{V}$ denotes the previously defined center of mass velocity, and $\mathbf{U}=\mathbf{v}_{1}-\mathbf{v}_{2}$ represents the diffusion velocity. The total stress

$$
\mathcal{T}=\mathcal{T}_{1}+\mathcal{T}_{2}-\left(1-\phi_{2}\right) \phi_{2} \mathbf{U} \otimes \mathbf{U}
$$

where $\mathcal{T}_{i}$ include the reversible and dissipative contributions to the stress.

The first two of the equations shown below follows by adding and subtracting, respectively, the equations of balance of linear momentum of the components (in the latter case, we first divide the individual equations by $\phi_{1}$ and $\phi_{2}$, respectively). The third equation is a form of the chain rule. The last equation is a consequence of (19). In addition, one of the three equations, (12), (13) or (17) has to be satisfied as well. We summarize these equations as follows,

$$
\begin{aligned}
& \frac{\partial \mathbf{V}}{\partial t}+(\mathbf{V} \cdot \nabla) \mathbf{V}=\nabla \cdot \mathcal{T} \\
& \frac{\partial \mathbf{U}}{\partial t}+\left(\phi_{2}-\phi_{1}\right)(\nabla \mathbf{U}) \mathbf{U}+(\mathbf{U} \otimes \mathbf{U}) \nabla \phi_{2}+(\nabla \mathbf{V}) \mathbf{U}+(\nabla \mathbf{U}) \mathbf{V} \\
& =\frac{1}{\phi_{1}} \nabla \cdot \mathcal{T}_{1}-\frac{1}{1-\phi_{1}} \nabla \cdot \mathcal{T}_{2}-\frac{\beta}{\phi_{1}\left(1-\phi_{1}\right)} \mathbf{U} \\
& \frac{\partial F}{\partial t}+\left(\mathbf{V}+\left(1-\phi_{1}\right) \mathbf{U}\right) \cdot \nabla F=\nabla\left(\mathbf{V}+\left(1-\phi_{1}\right) \mathbf{U}\right) F \\
& \nabla \cdot \mathbf{V}=0
\end{aligned}
$$

We note that the first equation gives the balance of linear momentum for the mixture, with $\mathcal{T}$ denoting the total stress. The second equation governing the evolution of the 
diffusive velocity $\mathbf{U}$ describes the microstructure of the system. These set of equations should be supplemented with initial and boundary conditions.

We now give the expressions of the stress tensors for the Newtonian and viscoelastic cases, respectively.

\section{Newtonian stress tensors.}

The equations for the stress tensors of the system in the case that, both, polymer and solvent admit Newtonian dissipation are

$$
\begin{array}{ll}
\mathcal{T}_{1}= & \phi_{1} \mathcal{H}(B)-\left(\Pi_{1}+p \phi_{1}\right)+\nu_{1}\left(\phi _ { 2 } ^ { - 2 } \left(\mathbf{V} \otimes \nabla \phi_{1}\right.\right. \\
& \left.\left.+\nabla \phi_{1} \otimes \mathbf{V}\right)+\phi_{2}^{-1}\left(\nabla \mathbf{V}+\nabla \mathbf{V}^{T}\right)+\left(\nabla \mathbf{U}+\nabla u^{T}\right)\right), \\
\mathcal{T}_{2}=\quad & -\left(\phi_{2} p+\Pi_{2}\right) I .
\end{array}
$$

The total stress now gives

$$
\begin{aligned}
\mathcal{T}=\quad & \phi_{1} \mathcal{H}(B)-\left(\Pi_{1}+\Pi_{2}+p\right) I \\
& +\left(\nu_{1}+\nu_{2}\right)\left(\phi_{2}^{-2}\left(\mathbf{V} \otimes \nabla \phi_{1}+\nabla \phi_{1} \otimes \mathbf{V}\right)+\phi_{2}^{-1}\left(\nabla \mathbf{V}+\nabla \mathbf{V}^{T}\right)\right. \\
& \left.\left.+\nu_{1}\left(\nabla \mathbf{U}+\nabla \mathbf{U}^{T}\right)\right)-\left(1-\phi_{2}\right) \phi_{2} \mathbf{U} \otimes \mathbf{U}\right) .
\end{aligned}
$$

\section{Viscoelastic stress tensors.}

In the case that the polymer response is viscoelastic with stress tensor as in (65), the total stress tensor, assuming Newtonian solvent with stress $\mathcal{T}_{2}$ as in $(74)$, is given by

$$
\begin{aligned}
\mathcal{T}= & \int_{-\infty}^{t}\left(2 \operatorname{det} B\left(t, t^{\prime}\right)^{-\frac{1}{2}}\left(\hat{w}_{I} B\left(t, t^{\prime}\right)+\hat{w}_{I_{2}}\left(I_{1} B\left(t, t^{\prime}\right)-B\left(t, t^{\prime}\right)^{2}\right)+I_{3} \hat{w}_{I_{3}} \mathbf{I}\right) d t^{\prime}\right. \\
& \left.+\nu_{2}\left(\phi_{2}^{-2}\left(\mathbf{V} \otimes \nabla \phi_{1}+\nabla \phi_{1} \otimes \mathbf{V}\right)+\phi_{2}^{-1}\left(\nabla \mathbf{V}+\nabla \mathbf{V}^{t}\right)\right)-\left(1-\phi_{2}\right) \phi_{2} \mathbf{U} \otimes \mathbf{U}\right) \\
& \left.-\left(\Pi_{1}+\Pi_{2}+p\right) I\right)
\end{aligned}
$$

\subsection{Special regimes}

Two distinctive regimes emerge according to the following observations.

\subsubsection{Purely diffusive regime}

If $\mathbf{V}(\mathbf{x}, t)=0$ at $\mathrm{t}=0$, and also $\mathbf{V}(\mathbf{x}, t)=0$ for $\mathbf{x} \in \Omega, t>0$, then there is a solution to the equations satisfying $\mathbf{V}=0$ for all time. In fact, setting $\mathbf{V}=0$ in equation (68), we obtain a first order equation for $p$ that can be solved by direct integration in terms of the remaining fields of the problem. The resulting problem involving the independent fields $\left\{\mathbf{U}, \phi_{1}, F\right\}$ is formulated as follows:

$$
\begin{aligned}
& \frac{\partial \mathbf{U}}{\partial t}+\left(\phi_{2}-\phi_{1}\right)(\nabla \mathbf{U}) \mathbf{U}+(\mathbf{U} \otimes \mathbf{U}) \nabla \phi_{2} \\
& =\frac{1}{\phi_{1}} \nabla \cdot \mathcal{T}_{1}-\frac{1}{1-\phi_{1}} \nabla \cdot \mathcal{T}_{2}-\frac{\beta}{\phi_{1}\left(1-\phi_{1}\right)} \mathbf{U}, \\
& \frac{\partial F}{\partial t}+\left(1-\phi_{1}\right) \mathbf{U} \cdot \nabla F=\nabla\left(\left(1-\phi_{1}\right) \mathbf{U}\right) F
\end{aligned}
$$


and either one of the balance laws (12), (13) or (17).

Diffusion equation. A special case of the former results when the inertia terms in (77) are neglected, giving

$$
\mathbf{U}=\beta^{-1} \phi_{1}\left(1-\phi_{1}\right)\left(\frac{1}{\phi_{1}} \nabla \cdot \mathcal{T}_{1}-\frac{1}{1-\phi_{1}} \nabla \cdot \mathcal{T}_{2}\right)
$$

A simple calculation shows that in the case $\mathbf{V}=0$, then $\mathbf{v}_{1}=\left(1-\phi_{1}\right) \mathbf{U}$ which substituted into equation (12) allows us to write it as

$$
\frac{\partial \phi_{1}}{\partial t}+\nabla \cdot\left(\phi_{1}\left(1-\phi_{1}\right) \mathbf{U}\right)=0
$$

Substitution of $\mathbf{U}$ from (79) into the latter yields

$$
\frac{\partial \phi_{1}}{\partial t}=-\nabla \cdot\left(\beta^{-1} \phi_{1}^{2}\left(1-\phi_{1}\right)^{2}\left[\frac{1}{\phi_{1}} \nabla \cdot \mathcal{T}_{1}-\frac{1}{1-\phi_{1}} \nabla \cdot \mathcal{T}_{2}\right]\right)=0
$$

We point out that the right hand side of the previous equation contains a term of the form $\nabla \cdot\left(\beta^{-1} \kappa\left(\phi_{1}, F, \nabla \mathbf{U}\right) \nabla \phi_{1}\right)$ (here the dependence on $\nabla \mathbf{U}$ holds for the special case that the polymer dissipative stress is Newtonian). Moreover, if one could further assert that the coefficient multiplying $\triangle \phi_{1}$ in such a term is positive, that is $\kappa>0$ then it would follow that (80) is a (nonlinear) diffusion equation for $\phi_{1}$. This is a model widely used in the studies of gels and known as the diffusion model. The quantity $\beta^{-1}$ corresponds to the diffusion constant $D$ used in characterization of gels [4]. The inequality $\kappa>0$
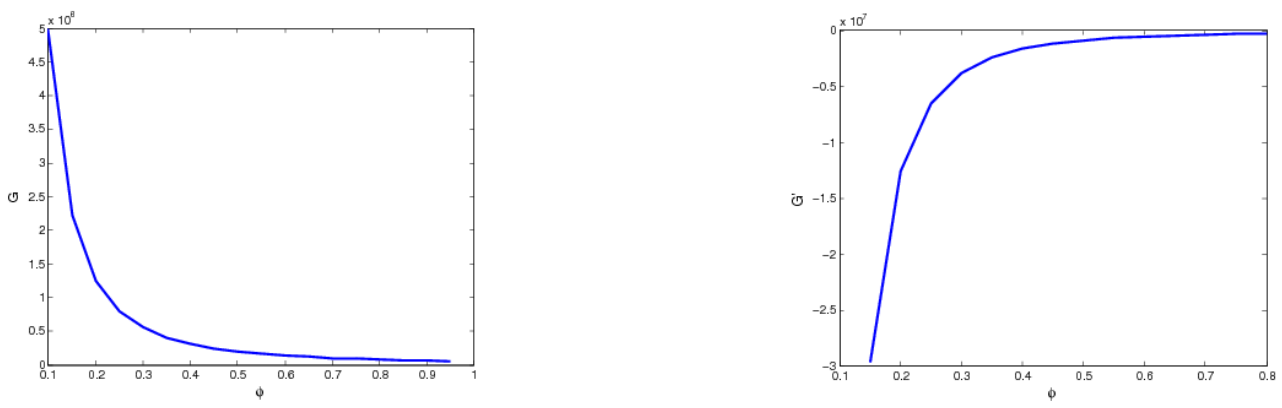

Figure 1: Graphs of $G(\phi)$ and $G^{\prime}(\phi)$ for polymer data: $a=.0001, b=1, c=.2, \mu_{0}=.5 * 10^{5}$. in general does not follow from thermodynamics. We appeal to the one-dimensional 
geometry to interpret such an inequality. The governing system (12) and (77) in the interval $(-1,1)$ in the one-dimensional geometry reduces to $[6]$

$$
\begin{aligned}
& \frac{\partial \phi_{1}}{\partial t}+\frac{\partial\left(\phi_{1}\left(1-\phi_{1}\right) U\right)}{\partial x}=0, \\
& \frac{\partial U}{\partial t}+\frac{\partial}{\partial x}\left(\frac{1}{2} U^{2}\left(1-2 \phi_{1}\right)-G\right)=-\frac{\beta}{\phi_{1}\left(1-\phi_{1}\right)} U,
\end{aligned}
$$

where $\mathbf{U}=(U(x, t), 0,0), \phi_{1}=\phi_{1}(x, t)$ and

$$
\begin{aligned}
& G\left(\phi_{1}\right)=\frac{K_{B} T}{V_{m} N_{x}}\left(-\frac{1}{2} \alpha^{2 / 3} \phi_{1}^{-\frac{2}{3}}-\left(\frac{1}{2}+\frac{N_{x}}{N_{1}}\right) \log \phi_{1}\right) \\
& +\mu \alpha^{2} \phi_{1}^{-2}-\frac{K_{B} T \chi}{V_{m}} \phi_{1}+\frac{K_{B} T}{N_{2} V_{m}} \log \left(1-\phi_{1}\right) .
\end{aligned}
$$

The analog to (79) obtained from (82) and its substitution into (81) yield

$$
\frac{\partial \phi_{1}}{\partial t}=-\frac{\left.\partial\left(\beta^{-1} G^{\prime}(\phi) \phi^{\prime}(x)\right)\right)}{\partial x} .
$$

The function $G(\phi)$ represents the extensional stress in terms of the polymer volume fraction. Equation (84) has a solution provided $G^{\prime}\left(\phi_{1}\right)<0$ holds. This monotonicity condition holds for the polymer data shown in section 3 . However, in the case of polysaccharides, there is a critical value $\phi_{c}=\phi_{c}(\mu), 0<\phi_{c}<1$ such that $G^{\prime}\left(\phi_{c}\right)=0$. Moreover, the value $\phi_{c}$ corresponds to a critical point of the volume phase transition between swollen and collapsed states [14]. Such properties are illustrated by the graphs of $G\left(\phi_{1}\right)$ and $G^{\prime}\left(\phi_{1}\right)$. The graphs in figure 1 correspond to polymer data with $\mu=2 * 10^{6} \frac{\mathrm{pN}}{\mathrm{nm}}$, whereas those in figure 2 are for polysaccharide data with $\mu=2 * 10^{-5} \frac{\mathrm{pN}}{\mathrm{nm} m^{2}}$. We observe the change of sign of $G^{\prime}\left(\phi_{1}\right)$ in the latter case. Later in this section, we will discuss the monotonicity condition from the dynamics point of view.
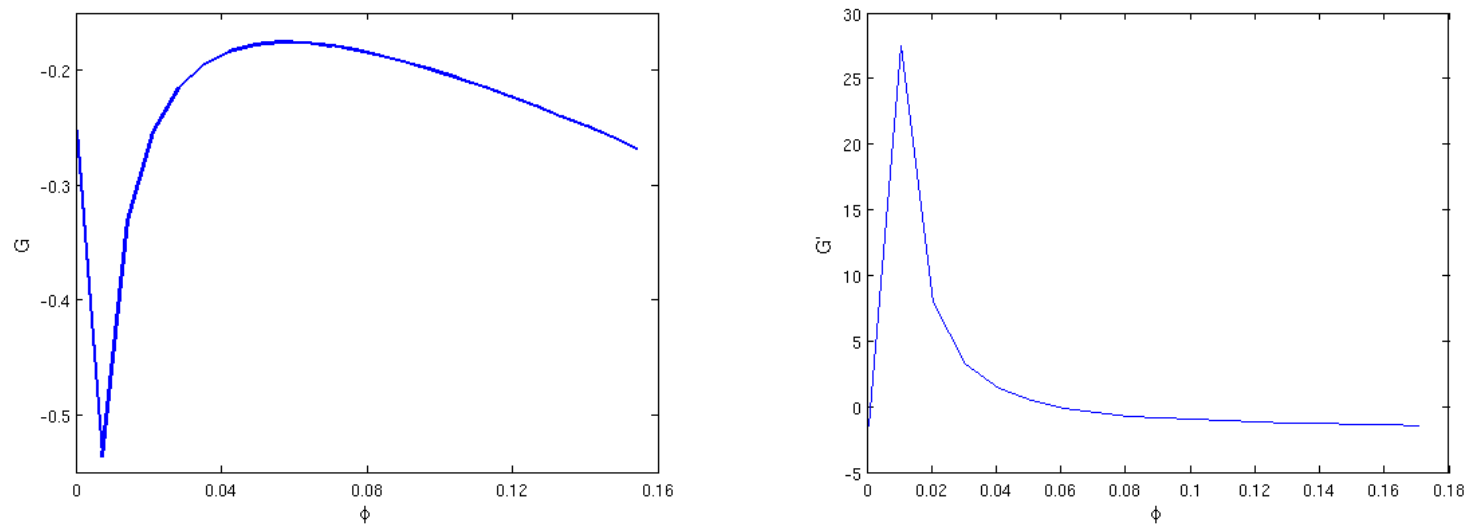

Figure 2: Graph of $G(\phi)$ and $G^{\prime}(\phi)$ for polysaccharide data: $a=.0001, b=1, c=.2, \mu_{0}=$ $.5 * 10^{-5}$. 


\subsubsection{Transport regime}

On the other hand, if $\mathbf{U}=0$ initially and on the boundary for all time, then the system becomes that of an incompressible viscoelastic fluid for the fields $\left\{\mathbf{V}, p, \phi_{1}, F\right\}$, with no diffusion present,

$$
\begin{aligned}
& \frac{\partial \mathbf{V}}{\partial t}+(\mathbf{V} \cdot \nabla) \mathbf{V}=\nabla \cdot \mathcal{T} \\
& \frac{\partial F}{\partial t}+\mathbf{V} \cdot \nabla F=(\nabla \mathbf{V}) F \\
& \nabla \cdot \mathbf{V}=0
\end{aligned}
$$

together with equations (19) and (17).

\subsection{Initial and boundary conditions}

On the boundary of the gel region, it is relevant to distinguish again between solid and solvent components of the material. We will present different types of boundary conditions motivated by applications. First of all, two main classes emerge according to whether the boundary is free, fixed or a combination of both types. For the sake of this presentation, we will assume that both, polymer has Newtonian stress and the solvent is inviscid (that is, $\eta_{1}=0$ in (63)). The governing system reduces then to equations (14), (15), (19), (17) and (16), together with constitutive equations (56), (57), (55), (40), (61), (37), (38), (53). The unknown fields of the problem are $\left\{\mathbf{v}_{1}, \mathbf{v}_{2}, F, p\right\}$. The governing system in the current domain $\Omega$ reduces to

$$
\begin{aligned}
& \dot{\mathbf{v}}_{1}=-\nabla p+\frac{\operatorname{det} F}{\phi_{0}}\left\{\nabla \cdot \mathcal{T}_{1}\left(F, \nabla \mathbf{v}_{1}\right)+\mathbf{f}_{1}\right\} \\
& \dot{\mathbf{v}}_{2}=-\nabla p+\frac{\operatorname{det} F}{\operatorname{det} F-\phi_{0}}\left\{\nabla \cdot \mathcal{T}_{2}(\operatorname{det} F)+\mathbf{f}_{2}\right\} \\
& \frac{\partial F}{\partial t}+\mathbf{v}_{1} \cdot \nabla F=\left(\nabla \mathbf{v}_{1}\right) F \\
& \nabla \cdot\left(\frac{\phi_{0}}{\operatorname{det} F} \mathbf{v}_{1}+\left(1-\frac{\phi_{0}}{\operatorname{det} F}\right) \mathbf{v}_{2}\right)=0 .
\end{aligned}
$$

To recover the deformation map of the polymer $\mathbf{x}=\mathbf{x}(\mathbf{X}, t)$, it is necessary to further solve the first order system

$$
\frac{\partial \mathbf{x}}{\partial \mathbf{X}}(\mathbf{X}, t)=F(\mathbf{x}(\mathbf{X}, t), t)
$$

in the reference domain $\Omega_{0}$.

\subsubsection{Gel confined to a given domain $\Omega$.}

This corresponds to the case that the polymer is chemically glued to the boundary $\partial \Omega$. This corresponds to requiring

$$
\mathbf{v}_{1}(\mathbf{x}, t)=0, \mathbf{x} \in \partial \Omega, t>0
$$


Another boundary condition is needed to characterize the degree of permeability of the membrane. Following Yamaue and Doi [25], [26], [27], (citeDOI04, we distinguish three type of membrane boundaries:

$$
\begin{aligned}
& \mathbf{v}_{2} \cdot \mathbf{n}=\mathbf{v}_{1} \cdot \mathbf{n}, \text { impermeable membrane } \\
& p(\mathbf{x}, t)+\Pi_{2}(\mathbf{x}, t)=P \text {, fully permeable membrane } \\
& P-\left(p(\mathbf{x}, t)+\Pi_{2}(\mathbf{x}, t)\right)=\kappa\left(\mathbf{v}_{2}-\mathbf{v}_{1}\right) \cdot \mathbf{n}, \text { semipermeable membrane, }
\end{aligned}
$$

where $P$ denotes the pressure of the external solvent, $\kappa$ is a permeability constant, and $\mathbf{n}$ is the unit normal vector to $\partial \Omega$. This type of boundary condition occurs in applications to body implantable biomedical devices where the polymer is glued to its support but the surface allows for penetrability of solvent. Since the polymer boundary is maintained fixed a build-up of stress occurs near the contact surface causing eventual failure of the device. The amount of normal stress acting on the boundary is that measured in the experiment by Suzuki and Hara [21] where the gel is confined between two circular disks with the bottom one maintained fixed and the top one allowed to move in order to relax the stress [25].

Initial data should be prescribed as well

$$
\mathbf{v}_{1}(\mathbf{x}, 0)=0, \mathbf{v}_{2}(\mathbf{x}, 0)=0 .
$$

In addition, we take the reference configuration to be $\Omega$ in which case $F=I$ at $t=0$, allowing for integration of (87).

\subsubsection{Gel subject to prescribed boundary force.}

Let us consider a gel with deformable boundary subject to a prescribed force. For instance, this is the case of the gel immersed in its own solvent subject to a constant pressure $P$. In addition to one of the three boundary conditions (92), (93) or (91) being prescribed, the boundary condition (90) is now replaced with

$$
-p \mathbf{n}+\left(\sigma_{1}+\sigma_{2}\right) \mathbf{n}=-P \mathbf{n}, \quad x \in \partial \Omega(t),
$$

where $\sigma_{1}$ and $\sigma_{2}$ are defined by (58). In the case that the membrane is permeable, combining (92) with (95) to eliminate $p$ gives the relation

$$
\Pi_{2} \mathbf{n}+\left(\sigma_{1}+\sigma_{2}\right) \mathbf{n}=0 .
$$

This relation involves the volume fraction at the interface. In order to study the motion of the free boundary with arbitrary geometry, it is necessary to employ the phase field or the level surface methods. For two special domains, one can directly supplement the previous boundary conditions with equations of evolution of the free boundary.

One-dimensional slab. We consider a gel initially confined in the strip $|x|<1$. Let $x=S(t)$ denote the location of the point initially at $x=1$ (by symmetry, $x=-S(t)$ describes the evolution of the left boundary). We assume that at time $t=0$, the polymer enters into contact with its own solvent, initially located at $|x|>1$. The evolution of the gel in the strip $|x| \leq S(t)$ is given by equations (81) and (82 for $\left\{\phi_{1}, U\right\}$, subject to initial and boundary conditions described next. The evolution equation of the interface between gel and pure solvent is given by

$$
\frac{d S(t)}{d t}=v_{1}(S(t), t), \quad S(0)=1 .
$$


Assuming that boundary conditions (95) and (92) hold, then (96) gives an equation for $\phi_{1}$ on the boundary $x=S(t)$. The value of $\phi^{*}$ that solves the equation is known as saturation volume fraction. We point out that such a value is only meaningful in onedimensional geometries since then $F$ and its determinant, det $F$ coincide. Moreover, one can then use the balance of mass relation $\phi_{1} \operatorname{det} F=\alpha$ to fully determine $\phi_{1}$ at the boundary from (96). This problem is analyzed in ([6]).

In particular, we find that the system is hyperbolic provided that the inequality $G^{\prime}\left(\phi_{1}\right)<0$ holds. This guarantees the propagation of the interface. As previously stated, such a condition holds for the polymer data shown in section 3 , and it can be interpreted as follows. A (dry) polymer slab brought into contact with its own solvent at the initial time, will keep swelling, with the speed of the interface decreasing with time. However, in the case of polysaccharides, there is a critical value $\phi_{c}=\phi_{c}(\mu), 0<\phi_{c}<1$ such that $G^{\prime}\left(\phi_{c}\right)=0$; if $\phi^{*}>\phi_{c}$, the interface will evolve, and the gel will continue swelling until $\phi_{1}$ reaches the critical value. The analysis suggests that $\phi_{c}$ may indicate the onset of de-swelling.

Spherical domain. In the case of a spherical domain (97) is represented by

$$
\frac{d r(t)}{d t}=v_{1}(r(t), t), \quad r(0)=R,
$$

where $R>0$ denotes the initial radius of the spherical gel region, and $v_{1}$ represents the

radial polymer velocity [6]. This case may be appropriate to the study of drug release devices.

\subsection{The stress-diffusion coupling model}

In this section, we present the quasi-static version of the previously developed model under a special choice of the free energy. This leads to the stress-diffusion coupling model proposed by Yamaue and Doi [26].

The assumption of no voids present in the material yields (72). Neglecting the FloryHuggins energy in (24), assuming the latter in the form of $\Psi=\phi_{1} \mu \operatorname{tr}\left(F^{T} F\right)$, the stress tensors in I (35) and (36), become

$$
\begin{aligned}
& \mathcal{T}_{1}=\phi_{1} \frac{\partial \psi_{1}}{\partial F} F^{T}-\phi_{1} p \\
& \mathcal{T}_{2}=-\phi_{2} p
\end{aligned}
$$

The corresponding system of balance laws of linear momentum is

$$
\begin{aligned}
& \phi_{1}\left(\frac{\partial \mathbf{v}_{1}}{\partial t}+\left(\mathbf{v}_{1} \cdot \nabla\right) \mathbf{v}_{1}\right)=\nabla \cdot\left(\phi_{1} \frac{\partial \psi_{1}}{\partial F} F^{T}\right)-\phi_{1} \nabla p-\beta\left(\mathbf{v}_{1}-\mathbf{v}_{2}\right) \\
& \phi_{2}\left(\frac{\partial \mathbf{v}_{2}}{\partial t}+\left(\mathbf{v}_{1} \cdot \nabla\right) \mathbf{v}_{2}\right)=-\phi_{2} \nabla p+\beta\left(\mathbf{v}_{1}-\mathbf{v}_{2}\right)
\end{aligned}
$$

Now, let us consider the equations of the steady state obtained by neglecting acceleration terms, that is, setting $\frac{\partial \mathbf{v}_{2}}{\partial t}+\left(\mathbf{v}_{2} \cdot \nabla\right) \mathbf{v}_{2}=0$ and $\frac{\partial \mathbf{v}_{1}}{\partial t}+\left(\mathbf{v}_{1} \cdot \nabla\right) \mathbf{v}_{1}=0$. Then the equations of balance of linear momentum reduce to

$$
\begin{aligned}
& \nabla \cdot \mathcal{T}_{1}+\mathbf{f}_{1}=0 \\
& \nabla \cdot \mathcal{T}_{2}+\mathbf{f}_{2}=0 .
\end{aligned}
$$


Since $\mathbf{f}_{1}=p \nabla \phi_{1}-\beta\left(\mathbf{v}_{1}-\mathbf{v}_{2}\right)(40)$, and $\mathbf{f}_{1}+\mathbf{f}_{2}=0$, the former become

$$
\begin{aligned}
& \nabla \cdot\left(\phi_{1} \frac{\partial W(F)}{\partial F} F^{T}\right)+\phi_{1} \nabla \cdot p-\beta\left(\mathbf{v}_{1}-\mathbf{v}_{2}\right)=0 \\
& -\phi_{2} \nabla p+\beta\left(\mathbf{v}_{1}-\mathbf{v}_{2}\right)=0
\end{aligned}
$$

and upon adding them, we obtain

$$
\nabla\left(\phi_{1} \frac{\partial W(F)}{\partial F} F^{T}-p\right)=0
$$

where $\frac{\partial W(F)}{\partial F}$ is the Piola-Kirchhoff stress tensor. Taking into account the balance of mass equation $\phi_{1} \operatorname{det} F=1$, we can rewrite (103) into

$$
\nabla\left(\operatorname{det} F^{-1} \frac{\partial W(F)}{\partial F} F^{T}-p\right)=0 .
$$

So, the corresponding stress tensor is given by the equation

$$
\sigma=\operatorname{det} F^{-1} \frac{\partial W(F)}{\partial F} F^{T}
$$

Summarizing, the resulting governing system is

$$
\begin{aligned}
& \nabla(\sigma-p I)=0 \\
& -\phi_{2} \nabla p+\beta\left(\mathbf{v}_{1}-\mathbf{v}_{2}\right)=0 \\
& \nabla \cdot\left(\left(1-\phi_{2}\right) \mathbf{v}_{1}+\phi_{2} \mathbf{v}_{2}\right)=0 \\
& \frac{\partial \phi_{2}}{\partial t}+\nabla \cdot\left(\phi_{2} \mathbf{v}_{2}\right)=0
\end{aligned}
$$

We observe that this system governs quasi-static regimes, with (102) corresponding to Darcy's law.

\subsubsection{Energy time rate and the Rayleghian}

Let us consider the two-component formulation given at the beginning of the article. Here we assume that only the laws of balance of mass hold and the constraint (4) hold. The goal is to provide an alternate derivation of the equations. Let us define the Rayleghian functional

$$
\begin{aligned}
R(t) & =\frac{d}{d t} \int_{\Omega(t)}\left(\frac{1}{2} \phi_{1} \mathbf{v}_{1} \cdot \mathbf{v}_{1}+\frac{1}{2} \phi_{2} \mathbf{v}_{2} \cdot \mathbf{v}_{2}+\Psi\right) d \mathbf{x} \\
& +\int_{\Omega(t)}\left(p \nabla \cdot\left(\phi_{1} \mathbf{v}_{1}+\phi_{2} \mathbf{v}_{2}\right)\right) d \mathbf{x}+W(t), \\
W(t) & =\frac{1}{2} \int_{\Omega(t)}\left(\beta\left(\phi_{1}, \phi_{2}\right)\left(\mathbf{v}_{1}-\mathbf{v}_{2}\right)^{2}\right) d \mathbf{x} \\
& +\int_{\partial \Omega(t)}\left(\left(\pi_{1}+\phi_{1} p\right) \mathbf{v}_{1}+\left(\pi_{2}+\phi_{2} p\right) \mathbf{v}_{2}\right) \cdot \mathbf{n}-\sigma \mathbf{n} \cdot \mathbf{v}_{1} \cdot d S
\end{aligned}
$$

The quantity $W(t)$ represents the power dissipated by the friction forces plus the that by the boundary forces. In addition, we have accounted for the fact that variations on 
$R(t)$ have to be carried out under the constraint $\nabla \cdot\left(\phi_{1} \mathbf{v}_{1}+\phi_{2} \mathbf{v}_{2}\right)=0$, and consequently, the power associated with maintaining the constraint is included. Let us compute the time derivative of the total energy.

$$
\frac{d}{d t} \mathcal{E}(t)=\int_{\Omega(t)}\left(\phi \mathbf{v}_{1} \cdot \dot{\mathbf{v}}_{1}+\phi_{2} \mathbf{v}_{2} \cdot \dot{\mathbf{v}}_{2}+\phi_{1} \dot{\psi}_{1}+\phi_{2} \dot{\psi}_{2}\right) d \mathbf{x}
$$

Now,

$$
\begin{aligned}
\phi_{1} \dot{\psi}_{1}+\phi_{2} \dot{\psi}_{2} & =\quad\left(\phi_{1} \frac{\partial \psi_{1}}{\partial \phi_{1}}+\phi_{2} \frac{\partial \psi_{2}}{\partial \phi_{1}}\right) \dot{\phi}_{1}+\left(\phi_{1} \frac{\partial \psi_{1}}{\partial \phi_{2}}+\frac{\partial \psi_{2}}{\partial \phi_{2}} \phi_{2}\right) \dot{\phi}_{2} \\
& +\quad \phi_{1} \frac{\partial \psi_{1}}{\partial F} \cdot \dot{F} \\
& =\quad-\pi_{1}\left(\nabla \cdot \mathbf{v}_{1}\right)-\pi_{2}\left(\nabla \cdot \mathbf{v}_{2}\right)+\phi_{1} \frac{\partial \psi_{1}}{\partial F} \cdot \dot{F} \\
& =\quad-\nabla \cdot\left(\pi_{1} \mathbf{v}_{1}\right)-\nabla \cdot\left(\pi_{2} \mathbf{v}_{2}\right) \\
& +\quad \nabla \pi_{1} \cdot \mathbf{v}_{1}+\nabla \pi_{2} \cdot \mathbf{v}_{2}+\phi_{1} \frac{\partial \psi_{1}}{\partial F} \cdot\left(\nabla \mathbf{v}_{1}\right) F
\end{aligned}
$$

where we have used (12) and (71). Using the definition of stress tensor,

$$
\sigma=\phi \frac{\partial \psi_{1}}{\partial F} F^{T}
$$

we rewrite

$$
\phi_{1} \frac{\partial \psi_{1}}{\partial F} \cdot\left(\nabla \mathbf{v}_{1}\right) F=\nabla \cdot\left(\sigma \mathbf{v}_{1}\right)-(\nabla \cdot \sigma) \cdot \mathbf{v}_{1} .
$$

Integrating and applying the divergence theorem, we get

$$
\begin{aligned}
\int_{\Omega(t)}\left(\phi_{1} \dot{\psi}_{1}+\phi_{2} \dot{\psi}_{2}\right) d \mathbf{x}=\quad & \int_{\Omega(t)}\left[\nabla \pi_{1} \cdot \mathbf{v}_{1}+\nabla \pi_{2} \cdot \mathbf{v}_{2}-(\nabla \cdot \sigma) \cdot \mathbf{v}_{1}\right] d \mathbf{x} \\
& -\int_{\partial \Omega}\left[\pi_{1} \mathbf{v}_{1} \cdot \mathbf{n}+\pi_{2} \mathbf{v}_{2} \cdot \mathbf{n}-\sigma \mathbf{n} \cdot \mathbf{v}_{1}\right] d S
\end{aligned}
$$

Also,

$$
\int_{\Omega(t)} p\left(\phi_{1} \nabla \cdot \mathbf{v}_{1}+\phi_{2} \nabla \cdot \mathbf{v}_{2}\right) \mathbf{x}=-\int_{\Omega(t)} \nabla p \cdot\left(\phi_{1} \mathbf{v}_{1}+\phi_{2} \mathbf{v}_{2}\right) d \mathbf{x}+\int_{\partial \Omega(t)} p\left(\phi_{1} \mathbf{v}_{1}+\phi_{2} \mathbf{v}_{2}\right) \cdot \mathbf{n} d S .
$$

Taking the previous calculations into account, we write

$$
\begin{aligned}
R(t)=\quad & \int_{\Omega(t)}\left(\left(\phi_{1} \dot{\mathbf{v}}_{1}-\nabla \cdot \sigma+\nabla \pi_{1}+\phi_{1} \nabla p\right) \cdot \mathbf{v}_{1}\right. \\
& \left.+\left(\phi_{2} \dot{\mathbf{v}}_{2}+\nabla \pi_{2}+\phi_{2} \nabla p\right) \cdot \mathbf{v}_{2}+\beta\left(\mathbf{v}_{1}-\mathbf{v}_{2}\right)^{2}\right) d \mathbf{x} .
\end{aligned}
$$

We now apply that variational principle of minimizing the Rayleghian with respect to $\mathbf{v}_{1}$ and $\mathbf{v}_{2}$ giving

$$
\begin{aligned}
& \phi_{1} \dot{\mathbf{v}}_{1}-\nabla \cdot \sigma+\nabla \pi_{1}+\phi_{1} \nabla p=\beta\left(\mathbf{v}_{1}-\mathbf{v}_{2}\right) \\
& \phi_{2} \dot{\mathbf{v}}_{2}+\nabla \pi_{2}+\phi_{2} \nabla p=-\beta\left(\mathbf{v}_{1}-\mathbf{v}_{2}\right) .
\end{aligned}
$$


We point out that the terms of the left hand side of the previous equations involving stress do not appear as perfect divergence. However adding the term $\phi_{1} \nabla p$ to both sides of the first equation, and likewise, the term $\phi_{2} \nabla$ to the second one give

$$
\begin{aligned}
& \phi_{1} \dot{\mathbf{v}}_{1}-\nabla \cdot \sigma+\nabla \pi_{1}+\nabla\left(\phi_{1} p\right)=\beta\left(\mathbf{v}_{1}-\mathbf{v}_{2}\right)+\phi_{1} \nabla p \\
& \phi_{2} \dot{\mathbf{v}}_{2}+\nabla \pi_{2}+\nabla\left(\phi_{2} p\right)=-\beta\left(\mathbf{v}_{1}-\mathbf{v}_{2}\right)+\phi_{2} \nabla p
\end{aligned}
$$

These now agree with the equations of balance of linear momentum with the stress and forcing terms obtained by imposing the Second Law of Thermodynamics.

\section{Conclusions}

We have derived a model of gel dynamics using the approach from mixture theory for a two-component system, polymer and solvent. The model allows for nonlinear elasticity, viscoelasticity, transport and diffusion. The governing equations consist of balance of mass and linear momentum for the individual components; the system is also formulated in terms of the center of mass velocity and the diffusive velocity, involving the total and the relative stresses. This allows for the identification of special regimes, such as purely the diffusive and purely transport ones. The total free energy of the system combines the elastic contribution of the polymer with the Flory-Huggins energy of mixing. We also obtain an equation for the rate of change of the total energy yielding decay for special choices of boundary conditions.

We appeal to the one-dimensional strip geometry to study special features of the dynamics. We focus on the early dynamics in contrast to the better understood relaxation studies; an scaling argument suggests that such a regime is well represented by the polymer-solvent friction. We find that the condition of propagation of the swelling interface, between the gel and the surrounding solvent, reduces to the monotonicity of the extensional stress (that is, the derivative of the stress as a function of the polymer volume fraction is negative, and it is positive with respect to the strain). Swelling in the case of the monotonic stress behavior, once started and with no other external effects, will continue up to the rest state. On the other hand, loss of monotonicity at some critical volume fraction hinders the swelling process. Indeed, we observed the monotonicity behavior in a linear entangled polymer, whereas we found loss of monotonicity in a polysaccharide. Specifically, we found an interval of volume fraction in a high swelling regime, where the stress changes monotonicity. This may suggest the onset of de-swelling or a volume phase transition taking place. The weak elasticity is responsible for the lose of monotonicity of the stress.

In the last part of the article, we carried out a comparison with other methods of derivation and models found in the literature, especially the stress-diffusion coupling model by Yamaue and Doi[26]. The latter emerged from earlier work by Doi and coauthors where the Rayleghian variational method was employed. We point out that derivations based on the Second Law of Thermodynamics, perhaps more complicated in development, have the advantage of determining the reversible contributions to the constitutive equations. 


\section{Acknowledgments}

The authors wish to thank Professor Masao Doi for the many discussions during his visit to the Institute for Mathematics and its Applications (IMA, University of Minnesota). The authors also wish to thank Professor Hans Weinberger for his many comments on the manuscript. This work is supported in part by Medtronic Inc, and by the National Science Foundation Grant number DMS-0456232.

\section{References}

[1] J. M. A.E. English, S. MAFe And ET.AL., Equilibrium swelling properties of polyampholytic hydrogels, J.Chem. Phys., 104 (1996), pp. 8713-8720.

[2] L. Bennethum and J. Cushman, Multiscale, hybrid mixture theory for swelling systems-i: Balance laws, Int. J. Eng. Sci, 34 (1996), pp. 125-145.

[3] — Multiscale, hybrid mixture theory for swelling systems-ii: Constitutive theory, Int. J. Eng. Sci, 34 (1996), pp. 147-169.

[4] R. B. Bird, R. C. Armstrong, and O. Hassager, Dynamics of Polymeric Liquids, vol. 1, John Wiley \& Sons, New York, 2 ed., 1987.

[5] D. K. C. Wolgemuth, E. Hoiczyk And G. Oster, How myxobacteria glide, Curr. Biol., 12 (2002), pp. 369-377.

[6] M. Calderer And H. Zhang, Incipient dynamics of swelling of gels, SIAM J. Appl. Math., to appear, (2008).

[7] C. Dafermos, Hyperbolic Conservation Laws in Continuum Physics, Springer, 2005.

[8] R. Diperna And P. Lions, Ordinary differential equations, transport theory and sobolev spaces, Inventiones Mathematicae, 98 (1989), pp. 511-547.

[9] M. DoI, Dynamics and Patterns in Complex Fluids, Springer, 1990.

[10] M. Doi And A. Onuki, Dynamic coupling between stress and composition in polymer solutions and blends, J. Phys. II France, 2 (1992), pp. 1631-1656.

[11] P. Flony, Principles of Polymer Chemistry, Cornell University Press, 1953.

[12] D. R. Gaskell, Introduction to the Thermodynamics of Materials, Taylor \& Francis, 1995.

[13] R. Larson, Constitutive Equations for Polymer Melts and Solutions, Butterworths, 1988.

[14] Y. Li And T. Tanaka, Phase transitions of gels, Ann. Rev. Mater. Sci., 22 (1992), pp. 243-77.

[15] F. Lin, C. LiU, And P. Zhang, On hydrodynamics of viscoelastic fluids, Comm. Pure Appl. Math., LVIII (2005), p. 1.

[16] C. Liu And N. J. Walkington, An eulerian description of fluids containing viscohyperelastic particles, Arch. Ration. Mech. Anal., 159 (2001), pp. 229-252.

[17] M. M. L.S. Bennethum And J. Cushman, Macroscale thermodynamics and the chemical potential of swelling porous media, Transport in Porous Media, 39 (2000), pp. 187-225. 
[18] C. Macosko, Rheology: Principles, Measurements and Applications, VCH Publishers, 1993.

[19] R. OGden, Non-Linear Elastic Deformations, Dover, 1997.

[20] A. OnUkI, Theory of pattern formation in gels: Surface folding in highly compressible elastic bodies, Phys Rev A, 39 (1989), pp. 5932-5948.

[21] A. Suzuki And T. HARA, Kinetics of one-dimensional swelling and shrinking of polymer gels under mechanical constraint, J.Chem.Phys., 114 (2001), p. 5012.

[22] T. Tanaka and D. Filmore, Kinetics of swelling of gels, J. Chem. Phys., 70 (1979), pp. 1214-1218.

[23] N. L. Thomas and A. H. Windle, A theory of case ii diffusion, Polymer, 23 (1982).

[24] C. Truesdell, Rational Thermodynamics, Springer Verlag, second edition, 1984.

[25] T. Yamaue And M. DoI, Swelling dynamics of constrained thin-plate gels under an external force, Phys. Rev. E, 70 (2004), p. 011401.

[26] _ Theory of one-dimensional swelling dynamics of polymer gels under mechanical constraint, Phys. Rev. E, 69 (2004), p. 041402.

[27] — The stress diffusion coupling in the swelling dynamics of cylindrical gels, J. Chem. Phys., 122 (2005), p. 084703.

[28] T. Yamaue, H. Mukai, K. Asaka, And M. Doi, Electrostress diffusion coupling model for polyelectrolyte gels, Macromolecules, 38 (2005), pp. 1349-1356. 